\title{
MICROSTRUCTURAL CONSIDERATIONS ON SH-WAVE PROPAGATION IN A PIEZOELECTRIC LAYERED STRUCTURE
}

\author{
Richa Goyal, Satish Kumar \\ School of Mathematics, Thapar Institute of Engineering and Technology, Patiala, Punjab, India \\ e-mail: richagoyal705@gmail.com; satishk.sharma@thapar.edu \\ VIKAS SHARMA \\ Department of Mathematics, Lovely Professional University, Phagwara, Punjab, India \\ e-mail: vikassharma10a@yahoo.co.in
}

\begin{abstract}
Shear wave based acoustic devices are being used in gaseous and liquid environments because of their high-sensitivity. The theoretical study of horizontally polarized shear (SH) waves in a layered structure consisting of a piezoelectric ceramic of $\mathrm{PZT}-5 \mathrm{H}$ or $\mathrm{BaTiO}_{3}$ material overlying a couple stress substrate is presented in this paper. The considered substrate is supposed to be exhibiting microstructural properties. The closed form expression of dispersion relations are derived analytically for electrically open and short conditions. The effects of internal microstructures of the couple stress substrate, thickness of $P Z T-5 H$ or $\mathrm{BaTiO}_{3}$ ceramic, piezoelectric and dielectric constants are illustrated graphically on the phase velocity of the piezoelectric layer under electrically open and short conditions.
\end{abstract}

Keyword: SH-wave, piezoelectricity, couple stress, characteristic length

\section{Introduction}

The propagation of surface waves in the layered system consisting of a finite layer having different material properties lying over a semi-infinite solid substrate has been of interest due to its great importance in geophysics, composite materials as well as in non-destructive evaluation. SH-waves refer to the type of surface waves which are horizontally polarized and propagate at the surface of the considered substrate. These waves exist only when a layer of finite thickness is deposited on semi-infinite substrate and the shear wave velocity in the layer is less than that of substrate. The piezoelectric material exhibits the linear coupling between mechanical and electric fields because of the ability of its material to produce an electric charge when subjected to mechanical stress and to produce deformation when subjected to an electric field. A thin film of piezoelectric material bonded over a solid substrate is used to improve the performance of surface acoustic wave (SAW) devices like sensors, transducer, resonators, filters, amplifiers, oscillators, delay lines etc. which are extremely or widely used in navigation, communication and in many other fields (Jakoby and Vellekoop, 1997).

Many researchers have investigated the piezoelectric layered structure to study propagation characteristics of SH-waves. Liu et al. (2001) investigated the effect of initial stress on the propagation behavior of Love waves in a layered piezoelectric structure. Wang (2002) examined shear horizontal (SH) wave propagation in a semi-infinite solid medium surface bonded by a layer of a piezoelectric material abutting the vacuum. Liu and He (2010) also illustrated the properties of Love waves for a layered structure with an $\mathrm{SiO}_{2}$ layer sputtered on an ST-90 $\mathrm{X}$ quartz substrate and found the existence of a threshold of normalized layer thickness. Wang et al. (2012) examined the dispersion behavior of SH waves propagating in a layered structure consisting of a piezoelectric layer and an elastic cylinder with an imperfect bonding. Wang and 
Zhao (2013) studied propagation of the Love wave in two-layered piezoelectric/elastic composite plates with an imperfect interface based on the shear spring model. Wei et al. (2009), Ezzin et al. (2017) examined the propagation of an SH-guided wave in the piezoelectric/piezomagnetic layered plates. Singh et al. (2015) investigated the propagation of a Love-type wave in an irregular piezoelectric layer lying over a piezoelectric half-space. Gupta and Vashishth (2016) studied Bulk wave propagation in a monoclinic porous piezoelectric material.

Though, Love or SH-wave propagation has been examined in detail for a piezoelectric layer overlying a solid substrate but the role of microstructure of the substrate has not been investigated to the full extent. The study of wave propagation in couple stress elastic space is of great interest due to its many applications, e.g. in polymers, cellular solids, composite materials and bones etc. Voigt (1887) proposed the idea of couple stress on the micro sized materials. The relevant mathematical model was presented by Cosserat and Cosserat (1909). Later, many researchers like Mindlin and Tiersten (1962), Koiter (1964), Eringen (1968) proposed different theories to explore this field further. The theories presented by these researchers carry certain drawbacks like indeterminacy of the spherical part of the couple-stress tensor and involvement of separate material length scale parameters. Hadjesfandiari and Dargush (2011) gave consistent couple stress theory which consisted of three material parameters $\lambda, \mu$ and characteristic length $(l)$ which described the effects of inner microstructure of the material. This characteristic length is negligible as compared to the dimensions of the body and is of the order of the average cell size or internal microstructure of the material. The propagation of $\mathrm{SH}$-waves are examined by various researchers under different conditions. Vardoulakis and Georgiadis (1997) examined the existence of SH surface waves in a homogeneous gradient-elastic half space with surface energy. Recently, co-authors Sharma and Kumar (2017) have investigated the propagation of $\mathrm{SH}$ waves in a viscoelastic layer bonded imperfectly with a couple stress substrate.

Thus, to enhance the domain of shear wave propagation, we intend to study the SH-wave propagation in a piezoelectric ceramic lying over a couple stress elastic half-space. The substrate is considered to have properties of a microstructure like granular macromorphic rock (Dionysos Marble). Two sets of a piezoelectric layer, i.e. $\mathrm{PZT}-5 \mathrm{H}$ and $\mathrm{BaTiO}_{3}$ materials are considered over a solid substrate. Closed form expressions of the dispersion equation for both the cases of electrically open and electrically short conditions for the propagation of SH-wave are obtained. Numerical computations are preformed for studying the effect of underlying microstructure of substrate, thickness of the layer, piezoelectric and dielectric constants on the phase velocity profiles of the shear wave.

\section{Formulation of the problem}

Here, we consider a piezoelectric layer of thickness $H$ (where $-H \leqslant x \leqslant 0$ ) lying over a couple stress elastic half-space. The Cartesian coordinate system is considered in such a way that the $\mathrm{SH}$-wave is propagating along the $y$-axis, and $x$-axis is pointing positive vertically downward as shown in Fig. 1. Conventionally, the poling direction is assumed along the $z$-axis.

If $u_{i}^{(p)}=\left(u_{1}, v_{1}, w_{1}\right)$ and $u_{i}^{(c)}=\left(u_{2}, v_{2}, w_{2}\right)$ are the mechanical displacement components due to propagation of the $\mathrm{SH}$-wave in the upper piezoelectric layer and the lower couple stress elastic half-space respectively. As the $\mathrm{SH}$-wave is propagating along the direction of the $y$-axis, this causes displacement in the $z$-direction only. We shall suppose that

$$
u_{i}=0 \quad v_{i}=0 \quad w_{i}=w_{i}(x, y, t) \quad(i=1,2)
$$

Let us suppose that the electric potential function of the upper piezoelectric layer is

$$
\phi=\phi(x, y, t)
$$




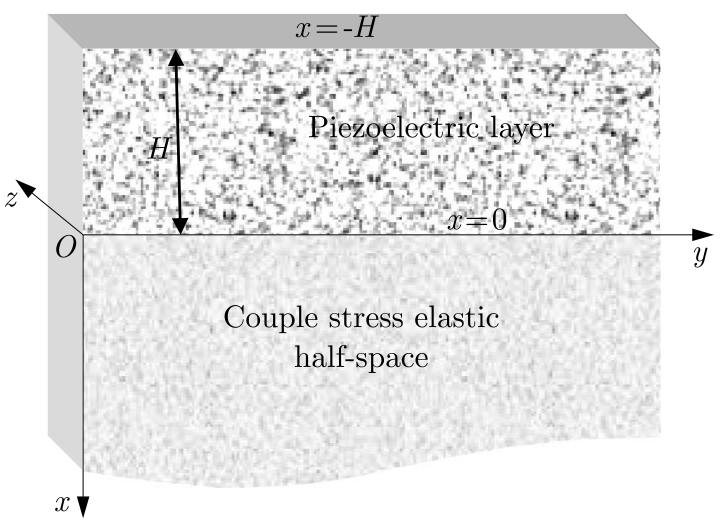

Fig. 1. Geometry of the problem

\subsection{Dynamics of the piezoelectric material layer}

The equation of motion for a piezoelectric layer, the electric displacement equilibrium equation and the constitutive relations may be written as

$$
\sigma_{i j, j}^{(p)}=\rho_{1} \frac{\partial^{2} u_{i}^{(p)}}{\partial t^{2}} \quad D_{i, i}=0
$$

and

$$
\sigma_{i j}^{(p)}=c_{i j k l} S_{k l}-e_{k i j} E_{k} \quad D_{j}=e_{j k l} S_{k l}+\epsilon_{j k} E_{k}
$$

where $i, j, k, l=1,2,3 ; \sigma_{i j}^{(p)}$ and $S_{k l}$ are the stress and strain tensors, respectively, $c_{i j k l}, e_{k i j}$ and $\epsilon_{j k}$ are the elastic, piezoelectric and dielectric coefficients respectively, $u_{i}^{(p)}=\left(u_{1}, v_{1}, w_{1}\right)$ and $D_{j}$ denotes the mechanical and electric displacement respectively, $E_{k}$ is the electrical potential field, $\rho_{1}$ is the mass density of the piezoelectric layer, the superscript index $p$ is used for the upper piezoelectric layer.

For the transversely isotropic piezoelectric layer, equation (2.4) can be expressed in the component form with the $z$-axis being the symmetric axis of the material as (Liu et al., 2001)

$$
\begin{array}{ll}
\sigma_{x}^{(p)}=c_{11} S_{x}+c_{12} S_{y}+c_{13} S_{z}-e_{31} E_{z} & \sigma_{y}^{(p)}=c_{12} S_{x}+c_{11} S_{y}+c_{13} S_{z}-e_{31} E_{z} \\
\sigma_{z}^{(p)}=c_{13} S_{x}+c_{13} S_{y}+c_{33} S_{z}-e_{33} E_{z} & \sigma_{y z}^{(p)}=c_{44} S_{y z}-e_{15} E_{y} \\
\sigma_{z x}^{(p)}=c_{44} S_{z x}-e_{15} E_{x} & \sigma_{x y}^{(p)}=\frac{1}{2}\left(c_{11}-c_{12}\right) S_{x y} \\
D_{x}=e_{15} S_{z x}+\epsilon_{11} E_{x} & D_{y}=e_{15} S_{y z}+\epsilon_{11} E_{y} \\
D_{z}=e_{31} S_{x}+e_{31} S_{y}+e_{33} S_{z}+\epsilon_{33} E_{z} &
\end{array}
$$

where $c_{11}, c_{12}, c_{13}, c_{33}, c_{44}$ are elastic constants, $e_{15}, e_{31}, e_{33}$ are piezoelectric constants and $\epsilon_{11}, \epsilon_{33}$ are dielectric constants. The relation between the strain components and mechanical displacement components as well as the relation between the electric potential field and the electric potential function as follows

$$
S_{i j}=\frac{1}{2}\left(u_{i, j}+u_{j, i}\right) \quad i, j=1,2,3 \quad E_{k}=-\phi_{, i}
$$

Using equation (2.1) in equation $(2.6)_{1}$ and equation (2.2) in equation $(2.6)_{2}$, we have

$$
\begin{array}{lcr}
S_{x}=0 & S_{y}=0 \quad S_{z}=0 & S_{x y}=0 \\
S_{y z}=\frac{\partial w_{1}}{\partial y} & S_{z x}=\frac{\partial w_{1}}{\partial x} &
\end{array}
$$


and

$$
E_{x}=-\frac{\partial \phi}{\partial x} \quad E_{y}=-\frac{\partial \phi}{\partial y} \quad E_{z}=0
$$

Now, substituting equation (2.7), (2.8) and (2.5), into equation (2.3), we can obtain the governing equations for the piezoelectric layer as

$$
c_{44} \nabla^{2} w_{1}+e_{15} \nabla^{2} \phi=\rho_{1} \ddot{w}_{1} \quad e_{15} \nabla^{2} w_{1}-\epsilon_{11} \nabla^{2} \phi=0
$$

Now, equations (2.9) take the form as

$$
\frac{\partial^{2} w_{1}}{\partial x^{2}}+\frac{\partial^{2} w_{1}}{\partial y^{2}}=\frac{1}{c_{p}^{2}} \frac{\partial^{2} w_{1}}{\partial t^{2}} \quad \frac{\partial^{2} \phi}{\partial x^{2}}+\frac{\partial^{2} \phi}{\partial y^{2}}=\frac{1}{c_{p}^{2}}\left(\frac{e_{15}}{\epsilon_{11}}\right) \frac{\partial^{2} w_{1}}{\partial t^{2}}
$$

where $c_{p}=\sqrt{c_{44}^{*} / \rho_{1}}, c_{44}^{*}=c_{44}+e_{15}^{2} / \epsilon_{11}$ and $c_{p}$ is the shear wave velocity in the piezoelectric layer.

We assume solutions to equations (2.10) in the form of

$$
w_{1}=W_{1}(x) \mathrm{e}^{\mathrm{i} \xi(y-c t)} \quad \phi=\varphi(x) \mathrm{e}^{\mathrm{i} \xi(y-c t)}
$$

where $\xi$ is the wave number and $c$ is the phase velocity. With the help of (2.10) and (2.11), it results in

$$
\begin{aligned}
& \frac{d^{2} W_{1}(x)}{d x^{2}}+\alpha^{2} \xi^{2} W_{1}(x)=0 \\
& \frac{d^{2} \varphi(x)}{d x^{2}}-\xi^{2} \varphi(x)+\frac{\xi^{2} c^{2}}{c_{p}^{2}} \frac{e_{15}}{\epsilon_{11}}\left[A_{1} \sin (\alpha \xi x)+A_{2} \cos (\alpha \xi x)\right]=0
\end{aligned}
$$

where $\alpha^{2}=\left(c^{2} / c_{p}^{2}\right)-1$.

Using the solutions to equations (2.11) and (2.12), we get solutions to equations (2.12) leads to

$$
\begin{aligned}
& w_{1}(x, y, t)=\left[A_{1} \sin (\alpha \xi x)+A_{2} \cos (\alpha \xi x)\right] \mathrm{e}^{\mathrm{i} \xi(y-c t)} \\
& \phi(x, y, t)=\left(\frac{e_{15}}{\epsilon_{11}}\left[A_{1} \sin (\alpha \xi x)+A_{2} \cos (\alpha \xi x)\right]+A_{3} \mathrm{e}^{-\xi x}+A_{4} \mathrm{e}^{\xi x}\right) \mathrm{e}^{\mathrm{i} \xi(y-c t)}
\end{aligned}
$$

where $A_{1}, A_{2}, A_{3}, A_{4}$ are arbitrary constants.

\subsection{Dynamics of couple stress elastic half-space}

The equation of motion for the couple stress elastic half-space in the absence of body forces and with the constitutive relations (Hadjesfandiari and Dargush, 2011) is

$$
\begin{aligned}
& \left(\lambda+\mu+\eta \nabla^{2}\right) \nabla\left(\nabla \cdot \mathbf{u}_{i}^{(c)}\right)+\left(\mu-\eta \nabla^{2}\right) \nabla^{2} \mathbf{u}_{i}^{(c)}=\rho_{2} \frac{\partial^{2} \mathbf{u}_{i}^{(c)}}{\partial t^{2}} \\
& \sigma_{j i}^{(c)}=\lambda u_{k, k} \delta_{i j}+\mu\left(u_{i, j}+u_{j, i}\right)-\eta \nabla^{2}\left(u_{i, j}-u_{j, i}\right) \\
& \mu_{j i}=4 \eta\left(\omega_{i, j}-\omega_{j, i}\right) \quad \text { where } \quad \omega_{i}=\frac{1}{2} \epsilon_{i j k} u_{k, j}
\end{aligned}
$$

where $i, j, k=1,2,3 ; \lambda, \mu$ are Cauchy-Lame constants, $\eta$ is the couple stress coefficient, $\eta=\mu l^{2}$ where $l$ is characteristic length, $\mathbf{u}_{i}^{(c)}=\left[u_{2}, v_{2}, w_{2}\right]$ is the displacement vector, $\rho_{2}$ is the mass density of the couple stress elastic half-space, $\sigma_{j i}^{(c)}$ is the non-symmetric force-stress tensor and 
$\mu_{j i}$ is the skew-symmetric couple stress tensor, $\delta_{i j}$ is Kronecker's delta and $\epsilon_{i j k}$ is the alternating tensor, the superscript index $c$ is used for the lower couple stress elastic half-space.

Using equation (2.1) in $(2.14)_{1}$, we have

$$
\frac{\partial^{2} w_{2}}{\partial x^{2}}+\frac{\partial^{2} w_{2}}{\partial y^{2}}-l^{2}\left(\frac{\partial^{4} w_{2}}{\partial x^{4}}+2 \frac{\partial^{4} w_{2}}{\partial x^{2} \partial y^{2}}+\frac{\partial^{4} w_{2}}{\partial y^{4}}\right)=\frac{1}{c_{1}^{2}} \frac{\partial^{2} w_{2}}{\partial t^{2}}
$$

where $c_{1}^{2}=\mu / \rho$ is the shear wave velocity in the couple stress substrate.

We assume the solution to equation (2.15) to be

$$
w_{2}=W_{2}(x) \mathrm{e}^{\mathrm{i} \xi(y-c t)}
$$

where $\xi$ is the wave number and $c$ is the phase velocity. Using this solution in equation (2.15), we get

$$
\frac{d^{4} W_{2}(x)}{d x^{4}}-S \frac{d^{2} W_{2}(x)}{d x^{2}}+T W_{2}(x)=0
$$

where

$$
S=2 \xi^{2}+\frac{1}{l^{2}} \quad T=\xi^{2}\left[\xi^{2}+\frac{1}{l^{2}}\left(1-\frac{c^{2}}{c_{1}^{2}}\right)\right]
$$

Since the amplitude of waves decreases with an increase in depth in the couple stress elastic half-space, so the solution to differential equation (2.17) becomes

$$
w_{2}(x, y, t)=\left(A_{5} \mathrm{e}^{-a x}+A_{6} \mathrm{e}^{-b x}\right) \mathrm{e}^{\mathrm{i} \xi(y-c t)}
$$

where

$$
a=\sqrt{\frac{S+\sqrt{S^{2}-4 T}}{2}} \quad b=\sqrt{\frac{S-\sqrt{S^{2}-4 T}}{2}}
$$

\section{Boundary conditions}

For propagation of SH-waves in a piezoelectric layer lying over a couple stress elastic half-space, the following boundary conditions are to be satisfied:

(A) Boundary conditions for the traction-free surface of the piezoelectric layer:

1. The mechanical stress-free condition is: $\sigma_{z x}^{(p)}=0$ at $x=-H$

2. The electrical boundary condition on the traction-free surface is:

(a) Electrically open condition: $D_{x}=0$ at $x=-H$

(b) Electrically short condition: $\phi_{p}=0$ at $x=-H$

(B) Boundary conditions at the common interface of the layer and half-space:

3. Stresses are continuous at the common interface: $\sigma_{z x}^{(p)}=\sigma_{z x}^{(c)}$ at $x=0$

4. Displacement fields are continuous at the common interface: $w_{1}=w_{2}$ at $x=0(3.5)$

5. Electric potential function should vanish at the common interface: $\phi_{p}=0$ at $x=0$

6. Couple stress tensor should vanish at the common interface: $\mu_{x y}=0$ at $x=0$ 


\section{Dispersion equations}

Using equations (2.13), (2.18) and their corresponding stress and electrical displacement components into boundary equations (3.1)-(3.7), we obtain the following algebraic equations in terms of unknown coefficients $A_{1}, A_{2}, A_{3}, A_{4}, A_{5}$ and $A_{6}$

$$
\begin{aligned}
& \alpha c_{44}^{*} \cos (\alpha \xi H) A_{1}+\alpha c_{44}^{*} \sin (\alpha \xi H) A_{2}-e_{15} \exp (\xi H) A_{3}+e_{15} \exp (-\xi H) A_{4}=0 \\
& \exp (\xi H) A_{3}-\exp (-\xi H) A_{4}=0 \\
& -\frac{e_{15}}{\epsilon_{11}} \sin (\alpha \xi H) A_{1}+\frac{e_{15}}{\epsilon_{11}} \cos (\alpha \xi H) A_{2}+\exp (\xi H) A_{3}+\exp (-\xi H) A_{4}=0 \\
& \alpha \xi c_{44}^{*} A_{1}-\xi e_{15} A_{3}+\xi e_{15} A_{4}+\mu a\left[1-l^{2}\left(\xi^{2}-a^{2}\right)\right] A_{5}+\mu b\left[1-l^{2}\left(\xi^{2}-b^{2}\right)\right] A_{6}=0 \\
& A_{2}-A_{5}-A_{6}=0 \\
& \frac{e_{15}}{\epsilon_{11}} A_{2}+A_{3}+A_{4}=0 \\
& \left(\xi^{2}-a^{2}\right) A_{5}+\left(\xi^{2}-b^{2}\right) A_{6}=0
\end{aligned}
$$

\subsection{Dispersion equations for the case of electrically open circuit}

The conditions mentioned in equations (4.1), (4.2) and (4.4)-(4.7) constitute six boundary conditions for this case. To obtain a non-trivial solution, the determinant of coefficients of the unknowns $A_{1}, A_{2}, A_{3}, A_{4}, A_{5}$ and $A_{6}$ vanishes. The frequency equation for the $\mathrm{SH}$-wave in an electrically open circuit is obtained as

$$
\xi\left(k_{a}-k_{b}\right)\left(\frac{e_{15}^{2}}{\epsilon_{11}} \tanh (\xi H)+\alpha c_{44}^{*} \tan (\alpha \xi H)\right)+\left[\mu a\left(1-l^{2} k_{a}\right) k_{b}-\mu b\left(1-l^{2} k_{b}\right) k_{a}\right]=0
$$

where $k_{a}=\xi^{2}-a^{2}$ and $k_{b}=\xi^{2}-b^{2}$.

Equation (4.8) represents dispersion relations of the SH-wave in an electrically open circuit for the piezoelectric layer lying over couple stress elastic half-space.

\subsection{Dispersion equations for the case of electrically short circuit}

The conditions mentioned in equations (4.1) and (4.3)-(4.7) constitute six boundary conditions for this case. To obtain a non-trivial solution, the determinant of coefficients of the unknowns $A_{1}, A_{2}, A_{3}, A_{4}, A_{5}$ and $A_{6}$ vanishes. The frequency equation for the SH-wave in an electrically short circuit is obtained as

$$
\begin{aligned}
& \xi\left(k_{a}-k_{b}\right)\left[\left(\alpha^{2} c_{44}^{* 2}-\frac{e_{15}^{4}}{\epsilon_{11}^{2}}\right) \tan (\alpha \xi H) \tanh (\xi H)+2 \alpha c_{44}^{*} \frac{e_{15}^{2}}{\epsilon_{11}}\left(1-\frac{\sec (\alpha \xi H)}{\cosh (\xi H)}\right)\right] \\
& +\left[\mu a\left(1-l^{2} k_{a}\right) k_{b}-\mu b\left(1-l^{2} k_{b}\right) k_{a}\right]\left(\alpha c_{44}^{*} \tanh (\xi H)-\frac{e_{15}^{2}}{\epsilon_{11}} \tan (\alpha \xi H)\right)=0
\end{aligned}
$$

Equation (4.9) represents dispersion relations of the SH-wave in an electrically short circuit for the piezoelectric layer lying over the couple stress elastic half-space.

\section{Numerical results}

For illustrating the results, we have considered a semi-infinite couple stress substrate which is made of Dionysos Marble having microstructural properties (Vardoulakis and Georgiadis, 1997): $\rho=2717 \mathrm{~kg} / \mathrm{m}^{3}, \mu=30.5 \cdot 10^{9} \mathrm{~N} / \mathrm{m}^{2}$ and $c_{p}=3350 \mathrm{~m} / \mathrm{s}$.

Piezoelectric layers of $\mathrm{PZT}-5 \mathrm{H}$ or $\mathrm{BaTiO}_{3}$ are considered (Liu et al., 2001) having properties as given below: 
(a) For PZT $-5 H$ ceramics: $c_{44}=2.30 \cdot 10^{10} \mathrm{~N} / \mathrm{m}^{2}, \rho=7.50 \cdot 10^{3} \mathrm{~kg} / \mathrm{m}^{3}, e_{15}=17.0 \mathrm{C} / \mathrm{m}^{2}$, $\epsilon_{11}=277.0 \cdot 10^{-10} \mathrm{C}^{2} / \mathrm{Nm}^{2}$

(b) For $\mathrm{BaTiO}_{3}$ ceramics: $c_{44}=4.40 \cdot 10^{10} \mathrm{~N} / \mathrm{m}^{2}, \rho=7.28 \cdot 10^{3} \mathrm{kKg} / \mathrm{m}^{3}, e_{15}=11.0 \mathrm{C} / \mathrm{m}^{2}$, $\epsilon_{11}=128.0 \cdot 10^{-10} \mathrm{C}^{2} / \mathrm{Nm}^{2}$

Dispersion curves of SH-type waves propagating in a piezoelectric layer overlying a couple stress medium have been examined in Figs. 2-9. Figures 2, 4, 6, 8 correspond to electrically open conditions and Figs. 3, 5, 7, 9 correspond to electrically short conditions. Phase velocity profiles are highly important for propagation of surface waves in the layered structure for its possible applications in sensors, delay lines, filters etc. One of the common feature of all theses characteristic curves is that the non-dimensional phase velocity $c / c_{p}$ decreases with an increase in the non-dimensional wave number $\xi H$.

\subsection{Effects of microstructure of the substrate}

Figures 2 and 3 show variation of the non-dimensional phase speed $c / c_{p}$ with respect to the non-dimensional wave number $\xi H$ for different values of characteristic length $l=0.00001 \mathrm{~m}$, $0.0001 \mathrm{~m}, 0.0004 \mathrm{~m}$. Here, the thickness of the piezoelectric layer is taken as $H=0.002 \mathrm{~m}$. It can be observed that the microstructure of the substrate affects the phase velocity profiles significantly. It can be seen from the profiles that with an increase in characteristic length, the phase velocity increases for both considered layers, i.e. for $P Z T-5 H$ material shown in 2(i) and 3(i) under electrically open conditions and for $\mathrm{BaTiO}_{3}$ material shown in 2(ii) and 3(ii) under electrically short conditions, as shown in Figs. 2 and 3, respectively. The characteristic curves clearly demonstrate microstructural effects of the semi-infinite solid substrate that remains ignored in the classical elastic model.
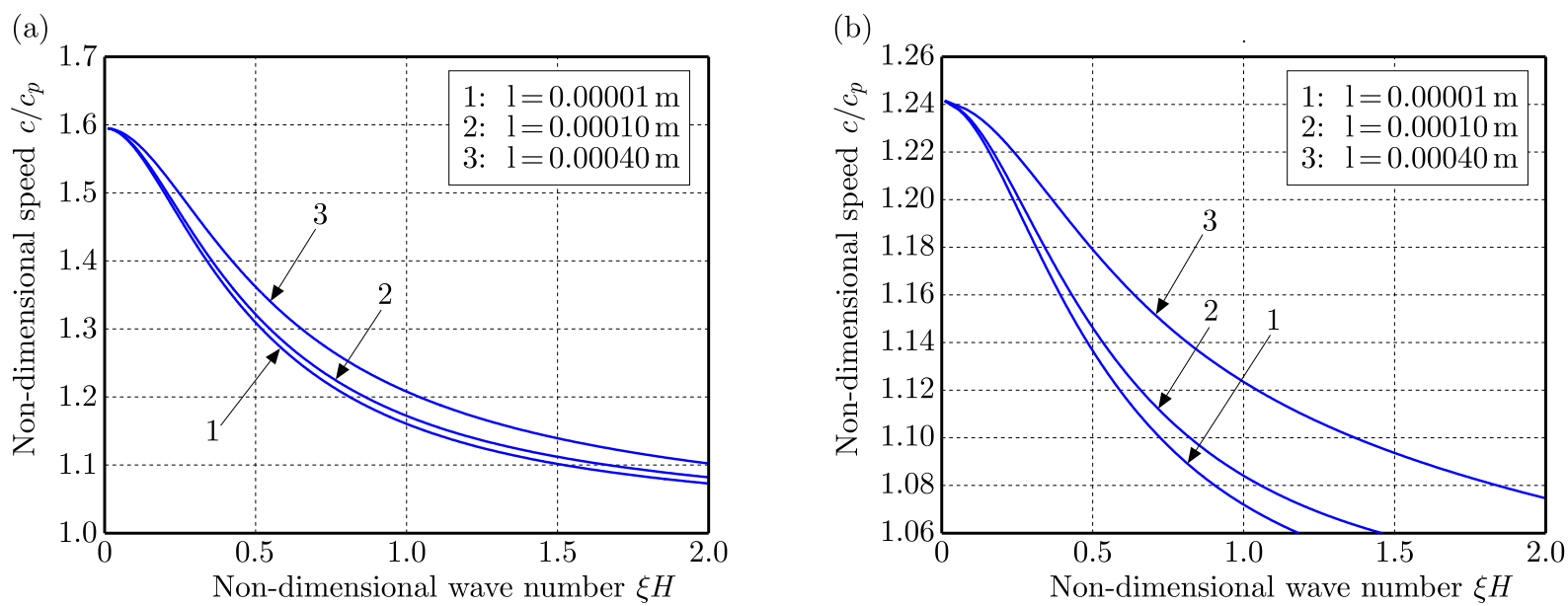

Fig. 2. Variation of the non-dimensional phase velocity against the non-dimensional wave number for different values of characteristic length $l=0.00001 \mathrm{~m}, 0.0001 \mathrm{~m}, 0.0004 \mathrm{~m}$, when $H=0.002 \mathrm{~m}$ in the case of electrically open conditions; (a) 2(i) for $\mathrm{PZT}-5 \mathrm{H}$ ceramic, (b) 2(ii) for $\mathrm{BaTiO}_{3}$ ceramic

\subsection{Effects of thickness of a piezoelectric layer}

To demonstrate the effects of thickness of a layer on the phase velocity profiles of the $\mathrm{SH}$-wave propagating in a layered structure, here in Figs. 4 and 5 we consider different values of the thickness of the layer i.e. $H=0.0005 \mathrm{~m}, 0.002 \mathrm{~m}, 0.05 \mathrm{~m}$, their characteristic length $l=0.0001 \mathrm{~m}$ is kept constant. It is observed that thickness of the layer has adverse effects on the phase velocities and the phase velocity decreases with an increase in thickness of the layer. 

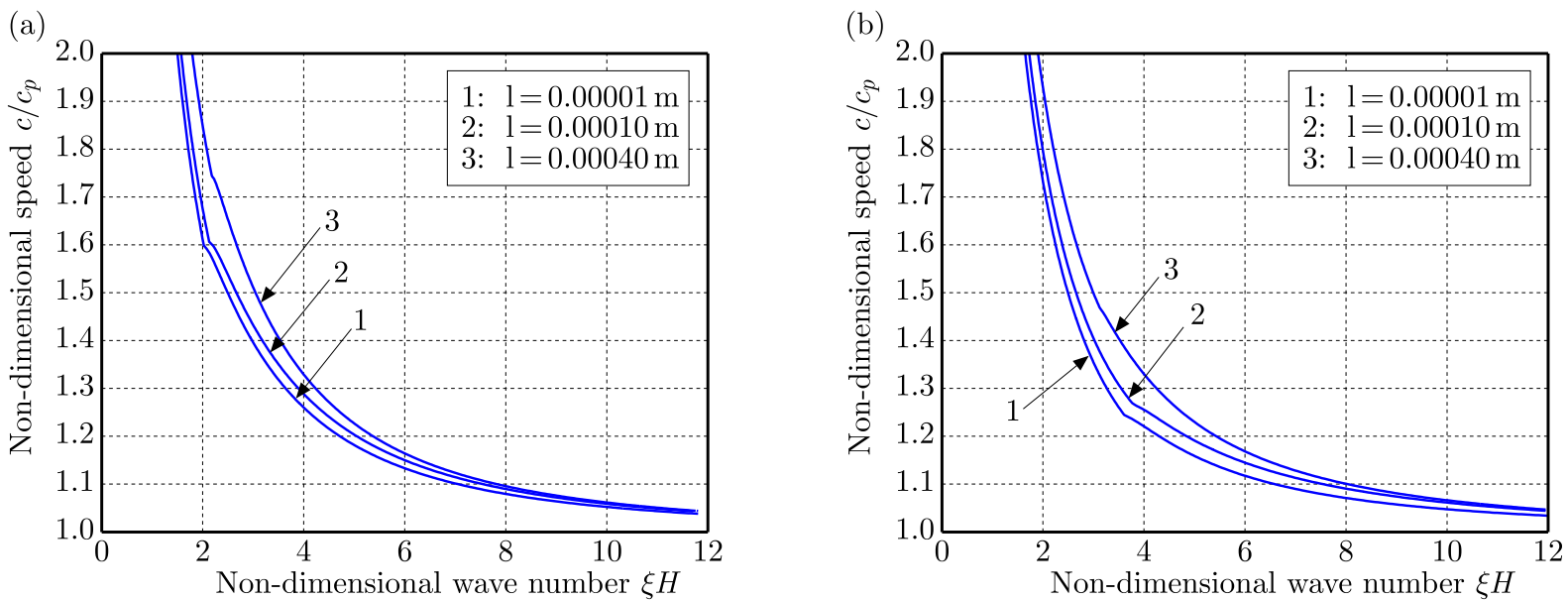

Fig. 3. Variation of the non-dimensional phase velocity against the non-dimensional wave number for different values of characteristic length $l=0.00001 \mathrm{~m}, 0.0001 \mathrm{~m}, 0.0004 \mathrm{~m}$, when $H=0.002 \mathrm{~m}$ in the case of electrically short conditions; (a) 3(i) for $\mathrm{PZT}-5 \mathrm{H}$ ceramic, (b) 3(ii) for $\mathrm{BaTiO}_{3}$ ceramic
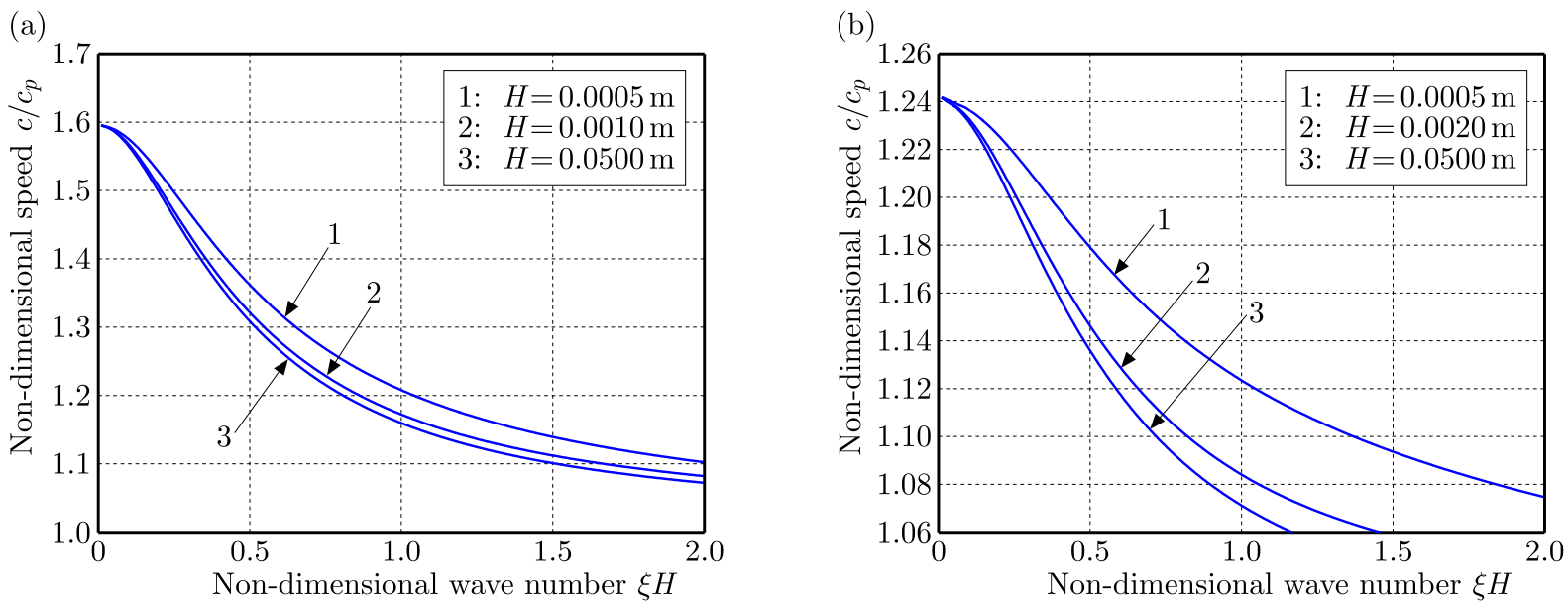

Fig. 4. Variation of the non-dimensional phase velocity against the non-dimensional wave number for different values of width of the piezoelectric layer $H=0.0005 \mathrm{~m}, 0.002 \mathrm{~m}, 0.05 \mathrm{~m}$, when $l=0.0001 \mathrm{~m}$ in the case of electrically open conditions; (a) 4(i) for $\mathrm{PZT}-5 \mathrm{H}$ ceramic, (b) 4(ii) for $\mathrm{BaTiO}_{3}$ ceramic
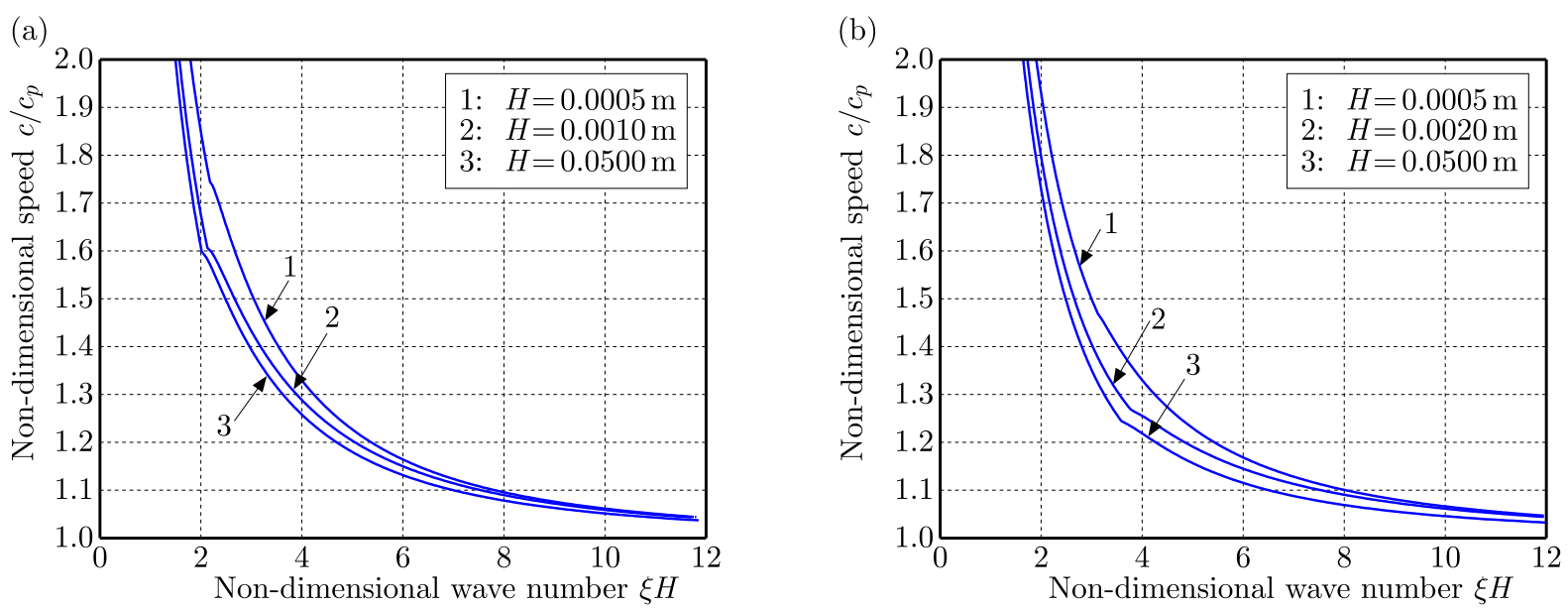

Fig. 5. Variation of the non-dimensional phase velocity against the non-dimensional wave number for different values of width of the piezoelectric layer $H=0.0005 \mathrm{~m}, 0.002 \mathrm{~m}, 0.05 \mathrm{~m}$, when $l=0.0001 \mathrm{~m}$ in the case of electrically short conditions; (a) 5 (i) for $\mathrm{PZT}-5 \mathrm{H}$ ceramic, (b) 5 (ii) for $\mathrm{BaTiO}_{3}$ ceramic 
Characteristic profiles for the corresponding electrically open and short conditions are shown in Figs. 4 and 5, 4(i) and 5(i) correspond to PZT - 5H material and 4(ii) and 5(ii) correspond to $\mathrm{BaTiO}_{3}$ material, respectively.

\subsection{Effects of piezoelectric constants}

Figures 6 and 7 show variation of the non-dimensional speed $c / c_{p}$ against the non-dimensional wave number $\xi H$ for the SH-wave propagation. The characteristic curves are plotted for different values of the piezoelectric parameter $e_{15}=17 \mathrm{C} / \mathrm{m}^{2}, 21 \mathrm{C} / \mathrm{m}^{2}, 25 \mathrm{C} / \mathrm{m}^{2}$ for $P Z T-5 H$ material shown in $6(\mathrm{i})$ and $7(\mathrm{i})$ and $e_{15}=11 \mathrm{C} / \mathrm{m}^{2}, 15 \mathrm{C} / \mathrm{m}^{2}, 19 \mathrm{C} / \mathrm{m}^{2}$ for $\mathrm{BaTiO} \mathrm{O}_{3}$ material shown in $6(\mathrm{ii})$ and 7 (ii) for both cases of electrically open and short conditions, respectively. The thickness of the layer $H=0.002 m$ and the characteristic length $l=0.0001 \mathrm{~m}$ associated with couple stress substrate are kept constant. The piezoelectric constant associated with the piezoelectric layer does not favor the phase velocity profiles of SH-waves. It is observed that an increase in the piezoelectric constant leads in general to a decrease in phase velocity profiles for both cases of electrically open and short conditions.
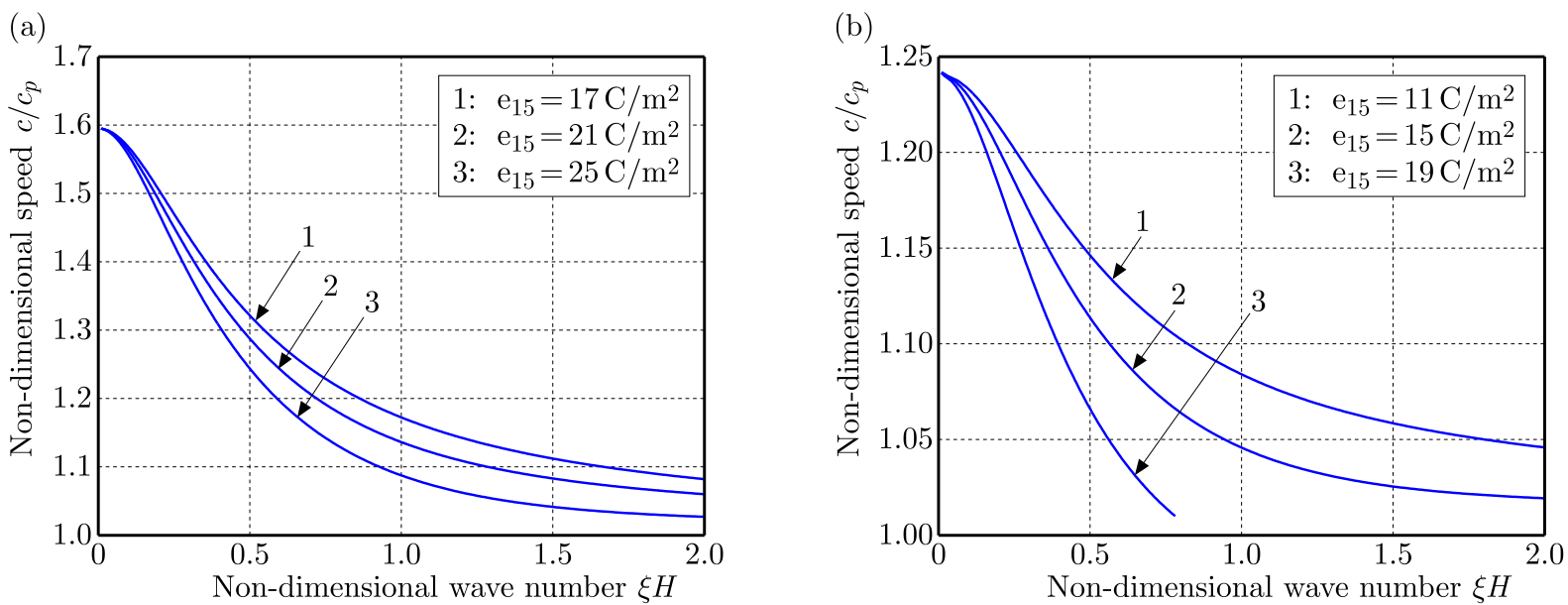

Fig. 6. Variation of the non-dimensional phase velocity against the non-dimensional wave number for different values of piezoelectric constants $e_{15}=17,21,25 \mathrm{C} / \mathrm{m}^{2}$ in $6(\mathrm{i})$ and $e_{15}=11,15,19 \mathrm{C} / \mathrm{m}^{2}$ in 6(ii), for electrically open cases; (a) 6(i) for $\mathrm{PZT}-5 \mathrm{H}$ ceramic, (b) 6(ii) for $\mathrm{BaTiO}_{3}$ ceramic
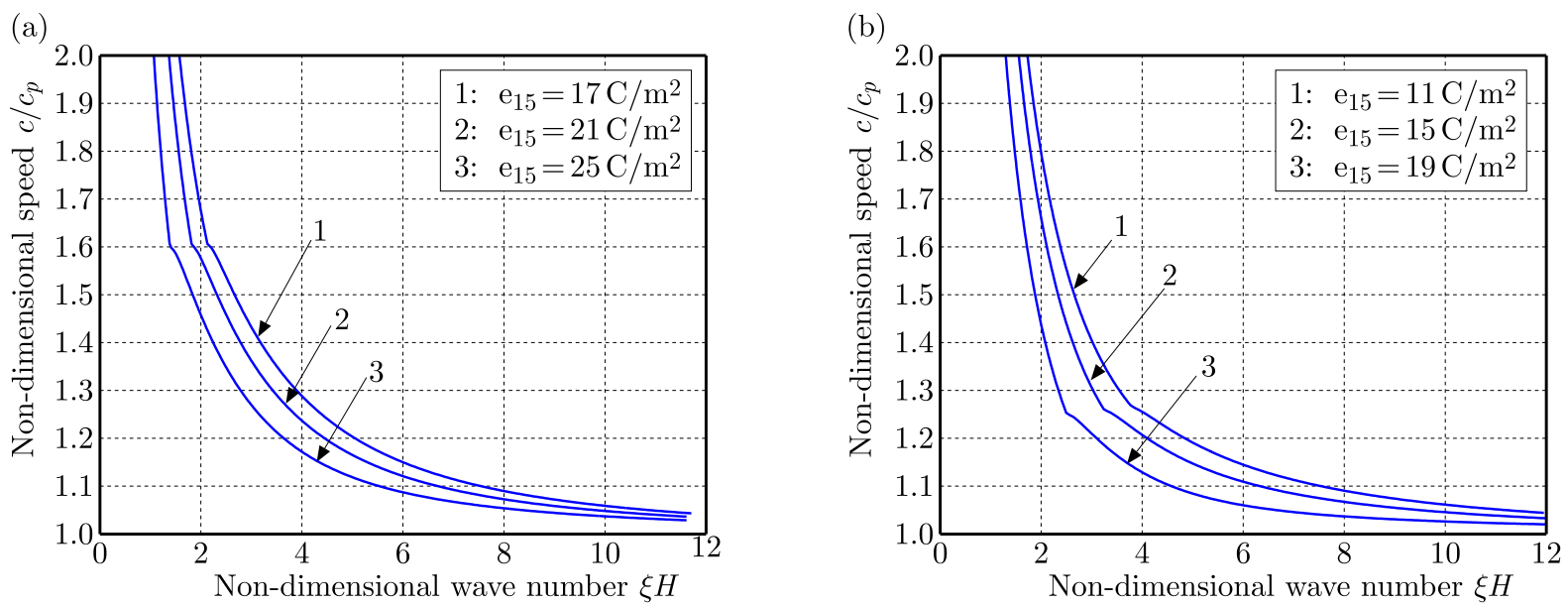

Fig. 7. Variation of the non-dimensional phase velocity against the non-dimensional wave number for different values of piezoelectric constants $e_{15}=17,21,25 \mathrm{C} / \mathrm{m}^{2}$ in $7(\mathrm{i})$ and $e_{15}=11,15,19 \mathrm{C} / \mathrm{m}^{2}$ in 7(ii), for electrically short cases; (a) 7(i) for $\mathrm{PZT}-5 \mathrm{H}$ ceramic, (b) 7(ii) for $\mathrm{BaTiO}_{3}$ ceramic 


\subsection{Effects of dielectric constants}

Figures 8 and 9 show the trend of the non-dimensional speed $c / c_{p}$ with respect to the non-dimensional wave number $\xi H$ on the $\mathrm{SH}$-type wave propagation for different values of dielectric constants $\epsilon_{11}=77 \mathrm{C}^{2} / \mathrm{Nm}^{2}, 177 \mathrm{C}^{2} / \mathrm{Nm}^{2}, 277 \mathrm{C}^{2} / \mathrm{Nm}^{2}$ for $P Z T-5 H$ material shown in $8(\mathrm{i})$ and $9(\mathrm{i})$ for electrically open conditions and $\epsilon_{11}=98 \mathrm{C}^{2} / \mathrm{Nm}^{2}, 128 \mathrm{C}^{2} / \mathrm{Nm}^{2}, 158 \mathrm{C}^{2} / \mathrm{Nm}^{2}$ for $\mathrm{BaTiO}_{3}$ material shown in 8 (ii) and 9 (ii) for electrically short conditions. Here, we have taken the material characteristic length parameter $l=0.0001 \mathrm{~m}$ and the thickness of the piezoelectric layer $H=0.002 \mathrm{~m}$. Dielectric constants of the piezoelectric layer overlying couple stress substrate affect the phase velocity profiles significantly. It is observed that the phase velocity of $\mathrm{SH}$-waves increases with an increase in the dielectric constant for both considered materials of the piezoelectric layer i.e. $\mathrm{PZT}-5 \mathrm{H}$ and $\mathrm{BaTiO}_{3}$ materials for both the cases of electrically open and short conditions.
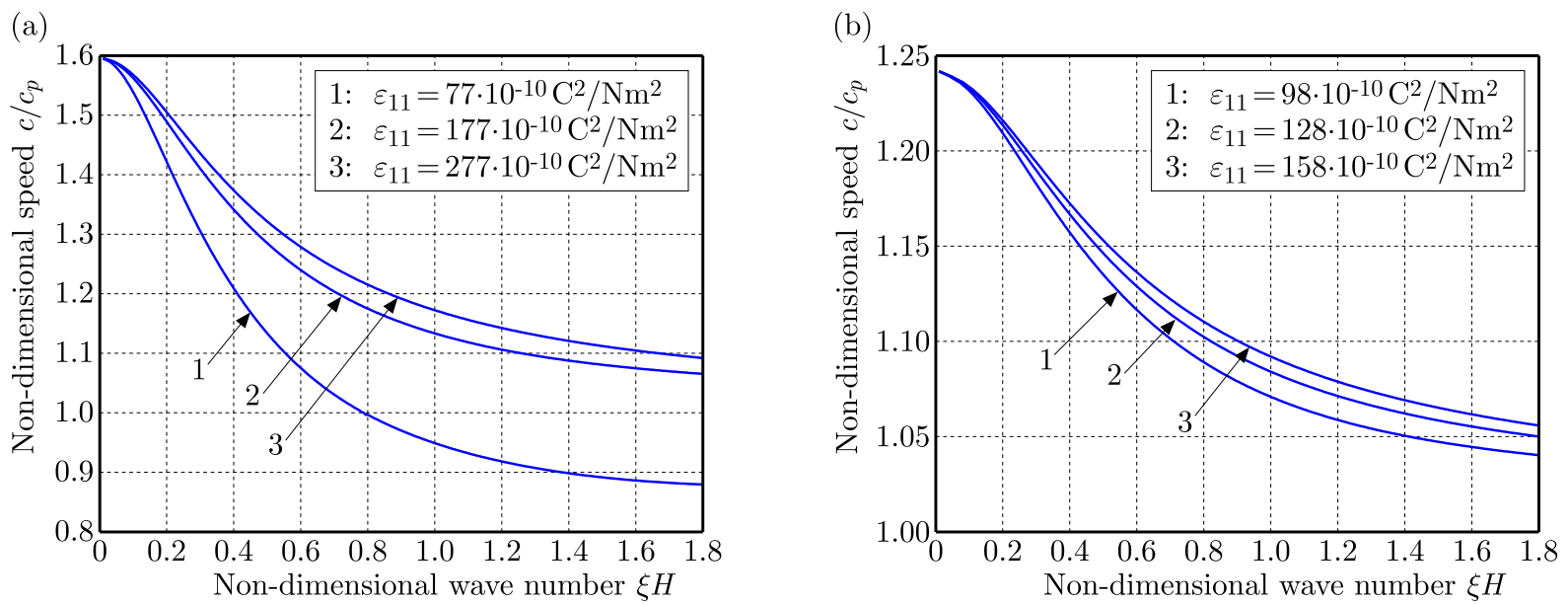

Fig. 8. Variation of the non-dimensional phase velocity against the non-dimensional wave number for different values of dielectric constants $\epsilon_{11}=77,177,277 \mathrm{C}^{2} / \mathrm{Nm}^{2}$ in $8(\mathrm{i}), \epsilon_{11}=98,128,158 \mathrm{C}^{2} / \mathrm{Nm}^{2}$ in 8(ii), for electrically open cases; (a) 8(i) for $\mathrm{PZT}-5 \mathrm{H}$ ceramic, (b) 8(ii) for $\mathrm{BaTiO}_{3}$ ceramic
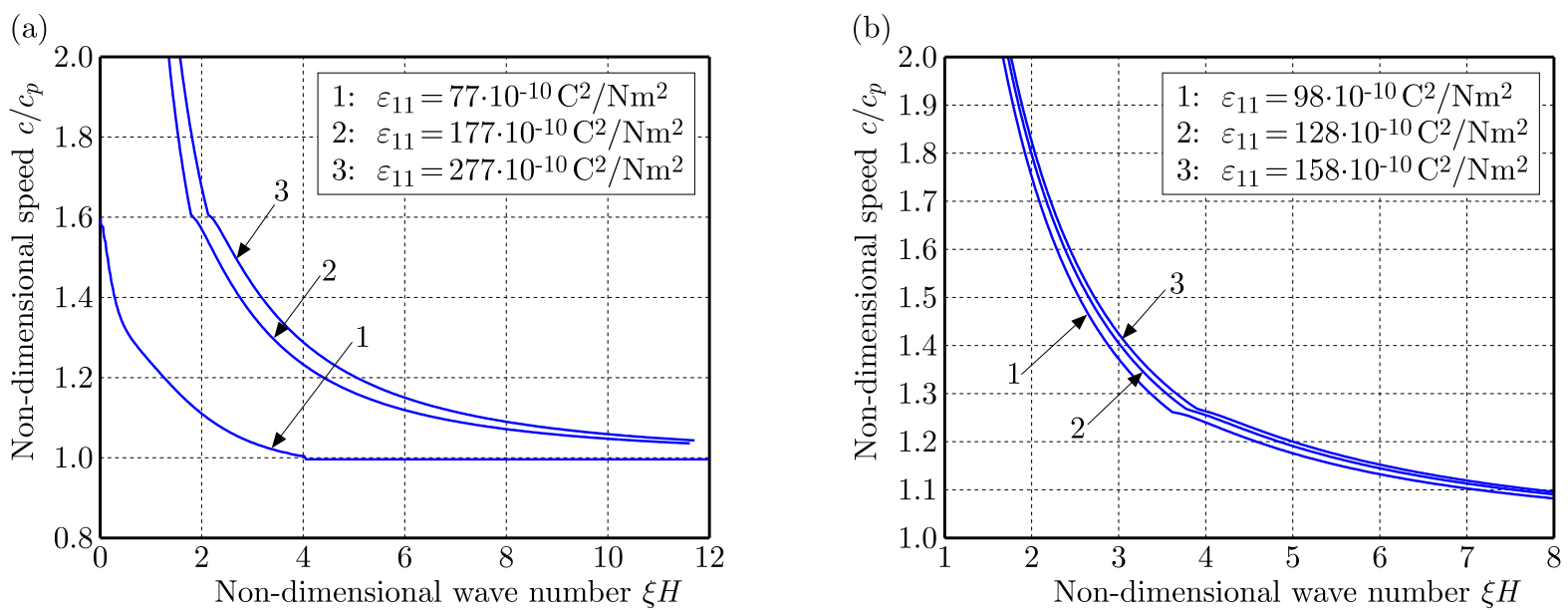

Fig. 9. Variation of the non-dimensional phase velocity against the non-dimensional wave number for different values of dielectric constants $\epsilon_{11}=77,177,277 \mathrm{C}^{2} / \mathrm{Nm}^{2}$ in $9(\mathrm{i}), \epsilon_{11}=98,128,158 \mathrm{C}^{2} / \mathrm{Nm}^{2}$ in

9 (ii), for electrically short case; (a) 9(i) for $\mathrm{PZT}-5 \mathrm{H}$ ceramic, (b) 9(ii) for $\mathrm{BaTiO}_{3}$ ceramic 


\section{Conclusion}

Dispersion equations (4.8) and (4.9) provide implicit relations between the phase velocity of SH-wave and different characteristic parameters associated with the layer and substrate. The phase velocity profiles are affected with variation in the associated parameters of the considered layered structure. The major conclusions of the study may be pointed out as follows:

(i) The wave number affects the phase velocity profiles of SH-waves significantly. The non-dimensional phase velocity decreases with an increase in the non-dimensional wave number in each case of electrically open and short circuits for both considered materials of the piezoelectric layer.

(ii) Internal microstructure of the couple stress substrate affects the phase velocity profiles significantly. It is observed that the phase velocity of SH-waves increases with an increase in the characteristic length $l$. This effect justifies consideration of microstructural properties of the semi-infinite substrate.

(iii) Thickness of the piezoelectric layer shows an adverse effect on phase velocity profiles of $\mathrm{SH}$-waves. It is observed that the phase velocity of SH-waves decreases with an increase in the thickness parameter associated with the piezoelectric layer.

(iv) The piezoelectric constant affects the phase velocity profiles of SH-wave substantially. Specifically, an increase in the piezoelectric parameter leads to a decrease in the phase velocity of $\mathrm{SH}$-waves propagation in the piezoelectric layer overlying the couple stress elastic half-space.

(v) Dielectric constants associated with the piezoelectric layer favor the phase velocity profiles of $\mathrm{SH}$-waves. It is observed that with an increase in the dielectric parameter, the phase velocity increases.

The findings obtained in the paper through theoretical and numerical demonstrations could help the development of more efficient and high performance Love wave based devices.

Acknowledgement

The authors gratefully acknowledge the support of the Indian government research agency: DST (Department of Science and Technology) via Grant No. EMR/2016/002601.

\section{References}

1. Cosserat E., Cosserat F., 1909, Theory of Deformable Bodies (in French), A Hermann et Fils, Paris

2. ERIngen A.C., 1968, Theory of micropolar elasticity, [In:] Fracture, H. Liebowitz (Edit.), Academic Press New York, 2, 662-729

3. Ezzin H., Amor M.B., Ghozlen M.H.B., 2017, Propagation behavior of SH waves in layered piezoelectric/piezomagnetic plates, Acta Mechanica, 228, 3, 1071-1081

4. Gupta V., Vashishth A.K., 2016, Effects of piezoelectricity on bulk waves in monoclinic poroelastic materials, Journal of Theoretical and Applied Mechanics, 54, 2, 571-577

5. Hadjesfandiari A.R., Dargush G.F., 2011, Couple stress theory for solids, International Journal of Solids and Structures, 48, 2496-2510

6. Jakoby B., Vellekoop M.J., 1997, Properties of Love waves: Applications in sensors, Smart Materials and Structures, 6, 668-679

7. Koiter W., 1964, Couple stresses in the theory of elasticity - I and II, Proceedings of the Koninklijke Nederlandse Akademie Van Wetenschappen, 67, 17-44 
8. LiU H., WAng Z.K., WAng T.J., 2001, Effect of initial stress on the propagation behavior of Love waves in a layered piezoelectric structure, International Journal of Solids and Structures, 38, $1,37-51$

9. LiU J., He S., 2010, Properties of Love waves in layered piezoelectric structures, International Journal of Solids and Structures, 47, 2, 169-174

10. Mindlin R.D., Tiersten H.F., 1962, Effects of couple-stresses in linear elasticity, Archive for Rational Mechanics and Analysis, 11, 415-448

11. Sharma V., Kumar S., 2017, Dispersion of SH waves in a viscoelastic layer imperfectly bonded with a couple stress substrate, Journal of Theoretical and Applied Mechanics, 55, 2, 535-546

12. Singh A.K., Kumar S., Chattopadhyay A., 2015, Love-type wave propagation in a piezoelectric structure with irregularity, International Journal of Engineering Science, 89, 35-60

13. Vardoulakis I., Georgiadis H.G., 1997, SH surface waves in a homogeneous gradient-elastic half-space with surface energy, Journal of Elasticity, 47, 2, 147-165

14. Voigt W., 1887, Theoretical Studies on the Elasticity Relationships of Crystals (in German), Abhandlungen der Gesellschaft der Wissenschaften zu Gttingen, 34

15. WANG H.M., ZHAO Z.C., 2013, Love waves in a two-layered piezoelectric/elastic composite plate with an imperfect interface, Archive of Applied Mechanics, 83, 1, 43-51

16. WANG Q., 2002, Wave propagation in a piezoelectric coupled solid medium, Journal of Applied Mechanics, 69, 6, 819-824

17. WAng Y., WAng M., LiU J., 2012, Propagation behaviors of SH waves in piezoelectric layer/elastic cylinder with an imperfect interface, Applied Mechanics and Materials, 151, 130-134

18. Wei W., LiU J., FAng D., 2009, Shear horizontal surface waves in a piezoelectric-piezomagnetic coupled layered half-space, International Journal of Nonlinear Sciences and Numerical Simulation, 10, 6, 767-778 